\title{
Fuzzy probabilistic data association filter and its application to single maneuvering target
}

\author{
Xiaobin $\mathrm{Li}^{1,2}$, En Fan ${ }^{3,4^{*}}$, Shigen Shen ${ }^{4,5}$, Keli Hu${ }^{4}$ and Pengfei $\mathrm{Li}^{6}$
}

\begin{abstract}
The fuzzy recursive least squares-probabilistic data association (FRLS-PDA) filter is presented for tracking single maneuvering target in cluttered situations with unknown process noises. In the proposed filter, the association probabilities of the current valid measurements belonging to a motion target are calculated by the probabilistic data association (PDA) algorithm. Then these probabilities are used to weight the valid measurements for generating a fused measurement, which is applied to determine the maneuvering characteristics of the moving target in real time including the current measurement residual and heading change. According to the above characteristics calculated, the fuzzy recursive least squares (FRLS) filter is used to estimate the current state of the target. The proposed filter can provide the advantage of the FRLS filter, which relaxes the restrictive assumptions of motion models of a maneuvering target. Moreover, it can realize single maneuvering target tracking in cluttered situations. The performance of the FRLS-PDA filter is evaluated by two experiments with the simulation data and real data, and it is found to be better than those of the PDA filter, IMM-PDA filter, fuzzy adaptive a- $\beta$ filter, and FRLS filter in tracking accuracy.
\end{abstract}

Keywords: Maneuvering target tracking, Probabilistic data association, Recursive least squares filter, Fuzzy system

\section{Introduction}

Due to the uncertainty in tracking process, single maneuvering target tracking (MTT) has become a difficult problem in information fusion, particularly in clutter [1, 2]. Generally, its tracking procedure can be divided into two steps: data association and state estimation. Data association originated from the uncertainty of sensor detection and observed environments, and state estimation is difficult to obtain due to the uncertainty of the motion model of a target. For tracking single maneuvering target, it needs to determine which measurements are from the target and then utilize the measurements to detect the maneuver and estimate the target state.

In the traditional data association methods, the nearest neighbor (NN) association algorithm is simple and practical one. It mainly utilizes the closest measurement to

\footnotetext{
* Correspondence: efan@usx.edu.cn

${ }^{3}$ ATR Key Laboratory, Shenzhen University, Shenzhen, Guangdong 518060, China

${ }^{4}$ Department of Computer Science and Engineering, Shaoxing University, Shaoxing, Zhejiang 312000, China

Full list of author information is available at the end of the article
}

update the filter, but may delete the possible measurement associated with the target [3]. The multiple hypothesis tracker (MHT) can establish the hypotheses of all possible measurements and keep these hypotheses [4]. Unfortunately, it easily leads to the exponential growth of association combinations. The probabilistic data association (PDA) algorithm is well accepted as the classical association method [5, 6]. It can delete the impossible hypotheses and establish the possible hypotheses of the associated measurements. Then these measurements are used to calculate the probabilities associated with the target. After data association, one needs to utilize the associated measurements to estimate a target's state. Many tracking methods based on the PDA algorithm have been successively developed [7, 8]. The interacting multiple model (IMM) algorithm is a wellknown filter for estimating the state of a maneuvering target, and its modified methods are broadly utilized in various applications [9-11]. The combination of the PDA algorithm and IMM algorithm is usually utilized in single maneuvering target tracking [12]. However, it is apt to bring incorrect tracking results when these tracking methods 
utilize mismatching sub-models generated by the uncertainty of motion models. Although the Kalman filter is usually used to track a target moving within a constant velocity, its tracking performance seriously degrades in tracking maneuvering targets. Moreover, it requires known process noises. A data-driven approach to tracking which has been presented in [13] uses the least squares fitting of a motion model to a segment of data. Considered process noises, the motion models fitted cannot exactly represent the real motion models of the target. The particle filter is wildly applied in various applications, particularly in maneuvering target tracking. An adaptive fuzzy particle filter (AFPF) method proposed in [14] is adapted to general object tracking in the field of computer vision. In the AFPF method, particle filtering samples are weighted using fuzzy membership functions and are applied to geometric and appearance features. The particle filter based on modified generalized probabilistic data association (PF-MGPDA) proposed in [15] combines the advantage of particle filter and generalized probabilistic data association to track maneuvering multi-targets. However, because the tracking performance of particle filter is combined in proportion to the number of the corresponding particles in target tracking, the maneuvering target tracking methods based on particle filter are difficult to satisfy the real tracking requirement of tracking systems. For this reason, here, the particle filter is not utilized to track maneuvering target in air surveillance. Although the $H_{\infty}$ filter and its modified forms have also been applied in MTT due to its main advantages on the insensitiveness to the exact knowledge of the external noise and more robustness to the parameter uncertainty, these filters need to assume the noise covariances are bounded, and this assumption is not suitable for high maneuver situations [16-18].

The mentioned methods above are in the framework of statistics theory, and they generally assume that the motion models of a maneuvering target are known. In real situations, the above requirement is usually difficult to satisfy [19-21]. Moreover, the real process noises and estimated errors are generally correlated, and meanwhile, there exists great uncertainty in tracking process. Based on these facts, the statistical association methods become complicated in real situations. Fuzzy theory can utilize the advantages of processing uncertainty information and it is generally utilized in state estimation [22, 23]. By incorporating fuzzy theory into the traditional tracking algorithms for solving the related uncertainty problems, it can improve the tracking performance of these tracking algorithms [24-26]. Combined the fuzzy PDA algorithm, the IMM fuzzy probabilistic data association algorithm (IMM-FPDA) proposed in [24] utilizes the prediction error and its change as the fuzzy inputs to optimize parameters of the fuzzy system, and the fuzzy system is used to determine the process noise covariance of the
Kalman filter. The hybrid fuzzy probabilistic data association filter (HF-PDAF) and hybrid fuzzy joint probabilistic data association filter [25] utilize a modified fuzzy cmeans (FCM) algorithm to reconstruct the membership grades between measurements to underlying tracks. The HF-PDAF method utilizes the Kalman filter to estimate the target's state, too. With the measurement residual, a new tracking algorithm proposed in [26] utilizes the FCM algorithm to extract the acceleration and estimate the motion of the target. Based on [26], a multiple-structured smart tracking algorithm developed in [27] separates the acceleration from the overall noise by fuzzy c-means clustering, and it can recognize the maneuvering target with multiple structures. As analyzed above, the Kalman filter can only be used in situations with known process noises. In situations of unknown process noises, the recursive least squares (RLS) filter can obtain the perfect estimated results and possess less calculation complexity compared with the traditional Kalman filter. It is widely applied in target tracking, system identification, and automatic control, etc. [28-31]. However, the RLS filter is only suitable to track a target moving at constant velocity. For this reason, the fuzzy recursive least squares (FRLS) filter is proposed for tracking single maneuvering target [28]. Unfortunately, it cannot be directly applied in cluttered situations.

Based on the above analysis, the fuzzy recursive least squares-probabilistic data association (FRLS-PDA) filter is proposed for single MTT in cluttered situations with unknown process noises. In the proposed filter, the PDA algorithm is applied to calculate the probabilities of the valid measurements belonging to the target. Then these probabilities are utilized to weight these measurements for constructing a fused measurement. According to the fused measurement, the measurement residual and heading change at current time are calculated, which act as the inputs of the fuzzy system designed while its output acts as the fuzzy fading factor of the FRLS filter. Hence, the proposed filter can estimate the current state of a maneuvering target through utilizing the fuzzy fading factor to adjust the influence of its predicted innovation on the predicted estimate. Furthermore, we analyze the adjustment function of the fuzzy fading factor on the predicted innovation for the current predicted state and then compare the calculation complexity of the proposed filter with those of the other three filters.

The remainder of this paper is organized as follows: in Section 2, the simplified form of the FRLS filter is deduced; in Section 3, the FRLS-PDA filter is proposed based on constructing a fused measurement, and both its fuzzy fading factor and computation complexity are analyzed; Section 4 presents the experiment results and the performance comparison with the PDA filter, IMM- 
PDA filter, fuzzy adaptive $\alpha-\beta$ filter, and FRLS filter; and finally, the conclusions are provided in Section 5.

\section{Fuzzy recursive least squares filter}

Assumed that a target moving with constant velocity at $k$ time, its motion and measurement model are generally expressed as follows (in the situation that there exists only a single target in air surveillance and the measurement associated with the target is determined):

$$
\begin{aligned}
& \boldsymbol{x}_{k+1}=\Phi_{k} \boldsymbol{x}_{k}+G_{k} \boldsymbol{v}_{k} . \\
& \boldsymbol{z}_{k}=H_{k} \boldsymbol{x}_{k}+\boldsymbol{w}_{k} .
\end{aligned}
$$

Here, $\boldsymbol{x}_{k}$ and $\boldsymbol{z}_{k}$ denote an $n$-dimensional state vector and an $m$-dimensional measurement vector, and $\Phi_{k}$ and $H_{k}$ denote an $n \times n$ state transition matrix and an $m \times n$ measurement transition matrix. $G_{k}$ is generally assumed to be a known matrix in $n \times n$ dimension. The process noise $v_{k}$ in $n \times 1$ dimension is assumed to be the zeromean Gaussian white noise with an $n \times n$ covariance $Q_{k}$, while the measurement noise $\boldsymbol{w}_{k}$ in $m \times 1$ dimension is assumed to be the zero-mean Gaussian noise with an $m \times m$ covariance $R_{k}$. Nevertheless, $Q_{k}$ and $R_{k}$ are usually unknown in real situations.

In [27], the FRLS filter is proposed to track single maneuvering target in uncluttered environments. Based on the recursive form of the FRLS filter, its simplified form is deduced as follows:

$$
\begin{aligned}
& \hat{\boldsymbol{x}}_{k}=\hat{\boldsymbol{x}}_{k-1}+K_{k} V_{k} . \\
& P_{k}=\tilde{\lambda}_{k}^{-1} P_{k-1}-K_{k} S_{k} K_{k}^{\mathrm{T}} .
\end{aligned}
$$

Here, $P_{k}$ is the $n \times n$ filter covariance matrix; $V_{k}, S_{k}$, $K_{k}$, and $\tilde{\lambda}_{k}\left(0<\tilde{\lambda}_{k} \leq 1\right)$ are respectively called the $n \times 1$ or predicted innovation, $n \times n$ residual covariance matrix, $n \times n$ gain matrix, and fuzzy fading factor, which can be, respectively, calculated by

$$
\begin{aligned}
V_{k} & =\boldsymbol{z}_{k}-H_{k} \Phi_{k} \hat{\boldsymbol{x}}_{k-1} . \\
S_{k} & =\tilde{\lambda}^{-1} H_{k} P_{k-1} H_{k}^{\mathrm{T}}+I . \\
K_{k} & =P_{k-1} H_{k}^{\mathrm{T}} S_{k^{-1}} . \\
\tilde{\lambda}_{k} & =\frac{\sum_{m=1}^{M} \bar{\lambda}_{l}^{m} \sup _{\gamma_{k} \in \tilde{A}_{i}^{m}, \phi_{k} \tilde{B}_{j}^{m}} \min \left(u_{\tilde{A}_{i}^{m}}\left(\gamma_{k}\right), u_{\tilde{B}_{j}^{m}}\left(\phi_{k}\right)\right)}{\sum_{m=1}^{M} \sup _{\gamma_{k} \in \tilde{A}_{i}^{m}, \phi_{k} \in \tilde{B}_{j}^{m}} \min \left(u_{\tilde{A}_{i}^{m}}\left(\gamma_{k}\right), u_{\tilde{B}_{j}^{m}}\left(\phi_{k}\right)\right)} .
\end{aligned}
$$

Here, $I$ is an $n \times n$ unit matrix, and $M=16$ denotes the number of fuzzy rules; $\gamma_{k}$ and $\phi_{k}$ are the measurement residual and heading change, which can be obtained by Eqs. (9) and (10); $\tilde{A}_{i}^{m}$ and $\tilde{B}_{j}^{m}$ denote the corresponding fuzzy sets of $\gamma_{k}$ and $\phi_{k}$ while $u_{\tilde{A}_{i}^{m}}$ and $u_{\tilde{B}_{i}^{m}}$ denote their membership degrees, respectively; $\tilde{C}_{l}^{m}$ and $u_{\tilde{C}^{m}}$ are assumed to be the fuzzy set and the membership function of $\tilde{\lambda}_{k} ; \bar{\lambda}_{l}^{m}$ is the corresponding value when $u_{\tilde{C}_{t}^{m}}$ obtains the maximal value in the $l$ th fuzzy set. In addition, all these notations above are also concretely explained in [27].

$$
\begin{aligned}
\gamma_{k} & =\left[V_{k}^{\mathrm{T}} V_{k}\right]^{1 / 2} / \gamma_{\max } \\
\phi_{k} & =\left|\varphi_{k}-\hat{\varphi}_{k-1}\right| / \phi_{\max } \\
\varphi_{k} & =\arctan \left[\left(y_{k}-\hat{y}_{k-1}\right) /\left(x_{k}-\hat{x}_{k-1}\right)\right] \\
\hat{\varphi}_{k-1} & =\arctan \left[\left(\hat{y}_{k-1}-\hat{y}_{k-2}\right) /\left(\hat{x}_{k-1}-\hat{x}_{k-2}\right)\right]
\end{aligned}
$$

where $\gamma_{\max }$ and $\phi_{\max }$ are the corresponding maximum values of the measurement residual and heading change; $V_{k}$ is the predicted innovation, which can be calculated by Eq. (5); $x_{k}, \hat{x}_{k-1}$, and $\hat{x}_{k-1}$ are the corresponding components of $\boldsymbol{z}_{k}, \hat{\boldsymbol{x}}_{k-1}$, and $\hat{\boldsymbol{x}}_{k-2}$ in the $x$-axis direction, while $y_{k}, \hat{y}_{k-1}$, and $\hat{y}_{k-2}$ are the components of $z_{k}, \hat{\boldsymbol{x}}_{k-1}$, and $\hat{\boldsymbol{x}}_{k-2}$ in the $\boldsymbol{y}$-axis direction.

Equations (3) and (4) are called the FRLS filter. Its initial states are determined with the measurements $z_{1}$ and $z_{2}$, given as follows:

$$
\begin{aligned}
& \hat{\boldsymbol{x}}_{2}=P_{2}\left[H_{1}^{\mathrm{T}}, H_{2}^{\mathrm{T}}\right]\left[\boldsymbol{z}_{1}^{\mathrm{T}}, \boldsymbol{z}_{2}^{\mathrm{T}}\right]^{\mathrm{T}} . \\
& P_{2}=\left(\left[H_{1}^{\mathrm{T}}, H_{2}^{\mathrm{T}}\right]\left[H_{1}^{\mathrm{T}}, H_{2}^{\mathrm{T}}\right]^{\mathrm{T}}\right)^{-1} .
\end{aligned}
$$

Here, both $H_{1}$ and $H_{2}$ are an $m \times m$ measurement transition matrix, and $P_{2}$ is an $n \times n$ filter covariance matrix.

\section{The proposed filter for MTT}

To track a single maneuvering target in clutter environments, this paper further proposes a FRLS-PDA filter based on the PDA algorithm and the FRLS filter. Its designed procedure mainly consists of three blocks, illustrated in Fig. 1. As shown in Fig. 1, firstly, the PDA algorithm is utilized to calculate the probabilities of the valid measurements associated with the target in block 1 ; secondly, these probabilities are used to weight the measurements for constructing a fused measurement in block 2; finally, one can calculate the measurement residual and the heading change according to the fused measurement in block 3 . Hence, the proposed filter can utilize the fuzzy system to determine the magnitude of the fading factor of the RLS filter, which can be used to adjust the influence of the predicted innovation on the current predicted state. 


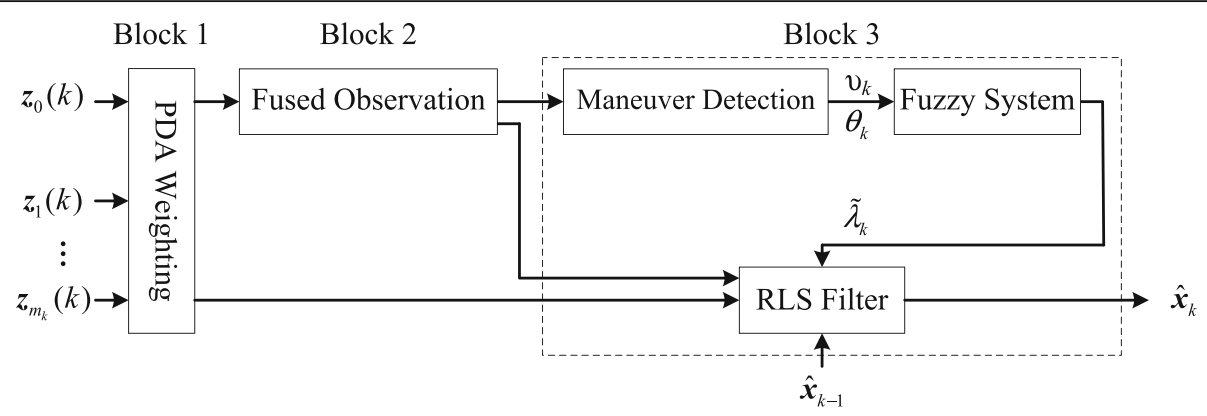

Fig. 1 Flow chart of FRLS-PDA filter

\subsection{Construct the fused measurement}

Assumed that the sets of the valid measurements and the cumulative measurement are, respectively, expressed by:

$$
\begin{aligned}
& \tilde{Z}(k)=\left\{\boldsymbol{z}_{i, k}\right\}_{i=1}^{m_{k}} \\
& \tilde{Z}^{k}=\{\tilde{Z}(j)\}_{j=1}^{k}
\end{aligned}
$$

where $z_{i, k}$ is the $i$ th valid measurement, and $m_{k}$ is the number of all valid measurements in the tracking gate at time $k$.

Based on the PDA algorithm, the state estimate of the target at time $k$ can be regarded as the probabilityweighted sum of local state estimates corresponding to all valid measurements. In the Poisson clutter model, the association probability on a measurement belonging to the target can be calculated by [3]:

$$
\beta_{i, k}=P\left\{\theta_{i, k} \mid \tilde{Z}^{k}\right\}=\mathrm{e}_{i} /\left(b+\sum_{j=1}^{m_{k}} \mathrm{e}_{j}\right), i=0,1, \cdots, m_{k}
$$

where

$$
\begin{aligned}
& \theta_{i, k}=\left\{\begin{array}{l}
\left\{z_{i, k} \text { belonging to the target }\right\} \quad i=1, \cdots, m_{k} \\
\text { no measurement belonging to the target }\} \\
i=0
\end{array}\right. \\
& \mathrm{e}_{0}=(2 \pi)^{\frac{M_{0}}{2}} \lambda \sqrt{\left|S_{i, k}\right|}\left(1-P_{D}\right) \\
& \mathrm{e}_{i}=P_{D} \exp \left[-\frac{1}{2} V_{i, k}^{\mathrm{T}} S_{i, k}^{-1} V_{i, k}\right]
\end{aligned}
$$

where $M_{\mathrm{o}}$ is the dimension of the measurement vector; $P_{D}$ is the detection probability; $V_{i, k}$ is the predicted innovation corresponding to $\boldsymbol{z}_{i, k}$, which can be calculated by Eq. (5); $S_{i, k}$ is the covariance matrix of $V_{i, k} ; \lambda$ is the parameter of Poisson distribution. If the clutter distribution is a uniform distribution, one only needs to reset the relative parameters [3].

In the PDA algorithm, the association probability may be used to evaluate the effect of the corresponding measurement in state estimation when there exist several measurements. Based on this fact, the influences of all valid measurements together on the current estimate are equal to that of a fused measurement. Hence, the fused measurement can be defined by

$$
\overline{\boldsymbol{z}}_{k}=\sum_{i=0}^{m_{k}} \beta_{i, k} \boldsymbol{z}_{i, k}
$$

Here, $\overline{\boldsymbol{z}}_{0, k}=H_{k} \hat{\boldsymbol{x}}_{k-1}$ is called as the zero measurement, which means that there are no measurements obtained. In this case, one utilizes the current position estimate as the current measurement.

\subsection{FRLS-PDA filter}

Based on the above conclusions, the main procedures of the FRLS-PDA filter can be described as below:

(1) Set the initial values $\hat{x}_{2}$ and $P_{2}$, and start the recursive formulas at $k=3$.

(2) Calculate the predicted innovation:

$$
V_{i, k}=\bar{z}_{i, k}-H_{k} \Phi_{k} \hat{x}_{k} .
$$

(3) Calculate the covariance of the predicted innovation:

$$
S_{k}=H_{k} P_{k-1} H_{k}+I .
$$

(4) Calculate the gain matrix:

$$
K_{k}=P_{k-1} H_{k} S_{k^{-1}} \text {. }
$$

(5) Calculate the tracking gate: $g^{2}$.

(6) Calculate the fading factor:

$$
\tilde{\lambda}_{k}=\frac{\sum_{m=1}^{M} \bar{\lambda}_{l}^{m} \sup _{\gamma_{k} \in \tilde{A}_{i}^{m}, \phi_{k} \in \tilde{B}_{j}^{m}} \min \left(\mu_{\tilde{A}_{i}^{m}}\left(\gamma_{k}\right), \mu_{\tilde{B}_{j}^{m}}\left(\phi_{k}\right)\right)}{\sum_{m=1}^{M} \sup _{\gamma_{k} \in \tilde{A}_{i}^{m}, \phi_{k} \in \tilde{B}_{j}^{m}} \min \left(\mu_{\tilde{A}_{i}^{m}}\left(\gamma_{k}\right), \mu_{\tilde{B}_{j}^{m}}\left(\phi_{k}\right)\right)} .
$$

(7) Calculate the FRLS filter: 


$$
\hat{x}_{k}=\hat{x}_{k \mid k-1}+K_{k} V_{k}
$$

where $V_{k}=\bar{z}_{k}-\hat{z}_{k \mid k-1}$.

(8) Update the filter covariance:

$$
\begin{aligned}
P_{k}= & \tilde{\lambda}_{k}^{-1} P_{k \mid k-1}-\tilde{\lambda}_{k}^{-2}\left[1-\beta_{0}\right] W_{k} S_{k} W_{k}^{\mathrm{T}} \\
& +\tilde{\lambda}_{k^{-2}} W_{k}\left[\sum_{i=1}^{m_{k}} \beta_{i, k} V_{i, k}, V_{i, k}^{\mathrm{T}}-V_{k} V_{k}^{\mathrm{T}}\right] W_{k}^{\mathrm{T}} .
\end{aligned}
$$

\subsection{Analysis of the fading factor and computation complexity}

In [28], the FRLS filter utilizes a measurement residual and a heading change as the inputs of the fuzzy system designed and its output as the fading factor of the FRLS filter. Then, the FRLS filter can adjust the influence of the predicted innovation on the current predicted state. In situations with unknown motion models, the FRLS filter can obtain good estimate results. Similarly, the FRLS-PDA filter can provide the above advantage of the FRLS filter. Furthermore, it can solve the single MTT problem in clutter by constructing a fused measurement according to all valid measurements at each time.

To better evaluate the performance of the MTT methods below, the computational complexity of the proposed filter is analyzed, which mainly denotes the times including multiplications, divisions, additions, and subtraction for each run of these filters under no consideration of the procedures of generating, moving, and measuring for a moving target. Here, it is assumed that the state vector and measurement vector are $n$-dimensional and $m$-dimensional, generally $m<n$. In addition, $l$ is the number of fuzzy rules utilized in Eq. (8), and $r$ is the number of sub-models in the IMM-PDA filter. The computational complexity for four types of filters is approximately given in Table 1 .

\section{Experiment results and analysis}

Two experiments of simulation data and real data have been carried out to evaluate the performance of the proposed filter in comparison with the other four filters, including the PDA filter [3], IMM-PDA filter [12], fuzzy adaptive (FA) $\alpha-\beta$ filter [21], and FRLS filter [28] in

Table 1 Computational complexity of four filters

\begin{tabular}{ll}
\hline Filter & Computational complexity \\
\hline PDA filter & $3 n^{3}+(6 m+1) n^{2}+\left(4 m^{2}+2 m-1\right) n+m^{3}+2 m$ \\
IMM-PDA filter & $3 n^{3}+(6 m+1) n^{2}+\left(4 m^{2}+2 m-1\right) n+2 m^{3}+2 m^{2}+$ \\
& $2 m+6 r$ \\
FRLS filter & $14 n^{3}+(4 m-1) n^{2}+\left(12 m^{2}+2 m-1\right) n+2 m^{3}-m^{2}+3 l$ \\
FRLS-PDA filter & $14 n^{3}+(4 m-1) n^{2}+\left(12 m^{2}+2 m-1\right) n+2 m^{3}+m^{2}+$ \\
& $2 m+3 l$ \\
\hline
\end{tabular}

terms of the position root mean squared errors (RMSE). In the experiments, the detection probability and gate probability set $P_{d}=1$ and $P_{g}=0.99$, respectively. The following experiments are conducted by using a computer with a dual-core $\mathrm{CPU}$ of Intel ${ }^{\circ} 2.20 \mathrm{GHz}, 8-\mathrm{GB}$ RAM. The programs are performed by using MATLAB 2009a version software.

\subsection{Simulation data experiment}

For easy comparison, we still adopt the similar simulation scenario in [28] but with clutter, concretely designed as follows. There exists a target moving in the air surveillance of 2 - D Cartesian $x y$-plane according to the given trajectory in Fig. 2. Its initial state is given by $\boldsymbol{x}_{0}=[800 \mathrm{~m}, 100 \mathrm{~m} / \mathrm{s}, 900 \mathrm{~m}, 173 \mathrm{~m} / \mathrm{s}]^{\mathrm{T}}$ and its trajectory is formed by 114 measurements collected by a radar located at $(0 \mathrm{~m}, 0 \mathrm{~m})$. The performance parameters of the radar are listed as follows: the sampling interval $T=$ $5 \mathrm{~s}$, range error $\sigma_{r}=15 \mathrm{~m}$, and azimuth error $\sigma_{\beta}=0.01^{\circ}$. The trajectory can be divided into five phrases: constant velocity motion, turn rate motion $(-0.0873 \mathrm{rad} / \mathrm{Ts})$, constant velocity motion, turn rate motion $(0.0873 \mathrm{rad} / T \mathrm{~s})$, and constant velocity motion. The turn model of the moving target is expressed as:

$$
\begin{aligned}
& \boldsymbol{x}_{k+1}=\left[\begin{array}{cccc}
1 & \frac{\sin \omega_{k} T}{\omega_{k}} & 0 & -\frac{1-\cos \omega_{k} T}{\omega_{k}} \\
0 & \cos \omega_{k} T & 0 & -\sin \omega_{k} T \\
0 & \frac{1-\cos \omega_{k} T}{\omega_{k}} & 1 & \frac{\sin \omega_{k} T}{\omega_{k}} \\
0 & \sin \omega_{k} T & 0 & \cos \omega_{k} T
\end{array}\right] \boldsymbol{x}_{k}+G_{k} \boldsymbol{v}_{k} \\
& G_{k}=\left[\begin{array}{cccc}
T^{2} / 2, & T, & 0, & 0 \\
0, & 0, & T^{2} / 2, & T
\end{array}\right]^{\mathrm{T}}
\end{aligned}
$$

where $\omega_{k}$ is the turn rate, and $\boldsymbol{v}(k)$ is the zero-mean white Gaussian noise with an unknown covariance. The clutter model is assumed to be of uniform distribution and the number of false measurements (clutters) is

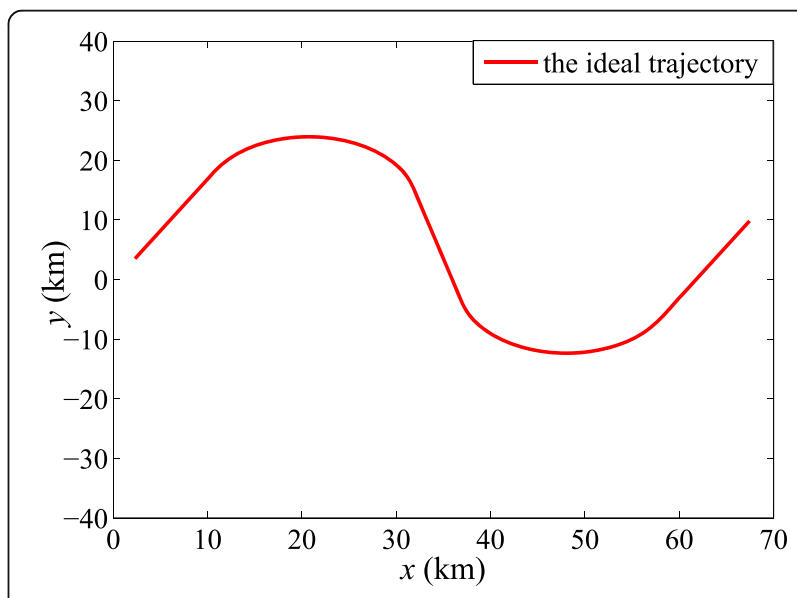

Fig. 2 Ideal target trajectory 


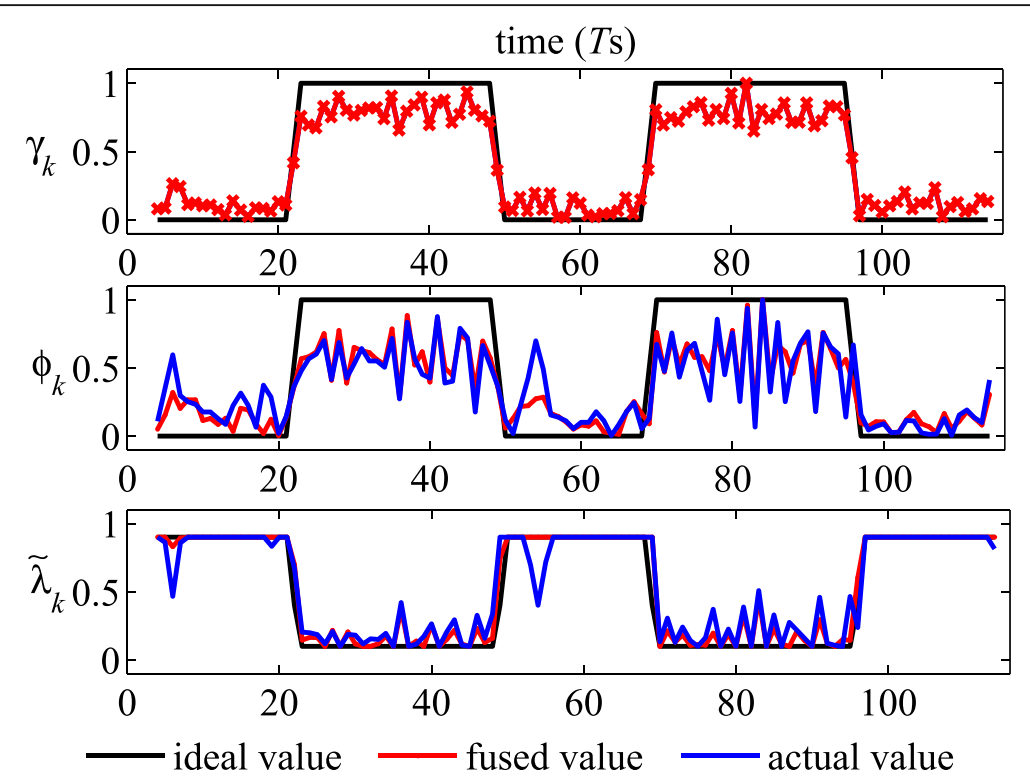

Fig. 3 Estimates of motion characteristics

assumed to be of Poisson distribution with known parameter $\lambda=10$ (the number of false measurements per unit of volume $\left(\mathrm{km}^{2}\right)$ ). To compare the performances of all filters, 100 Monte Carlo runs have been performed. In addition, the related information is assumed to satisfy the run requirements of the PDA filter, IMM-PDA filter, FA $\alpha-\beta$ filter, and FRLS filter. Because the particle filter generally consumes large time, it is difficult to apply in tracking system with high requirements in time and its comparison is not given here.

Figure 3 shows that the variation curves of $\gamma_{k}, \phi_{k}$, and $\tilde{\lambda}_{k}$ are obtained according to the target motion in the ideal, uncluttered, and cluttered case, respectively. Figures 4 and 5 provide the tracking results and the root-mean-square (RMS) position errors using the mentioned filters above. From Fig. 3, the magnitudes of their fading factors are close to the values obtained in the ideal case, and it is verified that the curves of $\gamma_{k}$ and $\phi_{k}$ in the uncluttered and cluttered case can reflect the maneuver changes exactly. From Figs. 4 and 5, the RMS position errors of the FRLS-PDA filter are smaller than the other four filters, concretely discussed as follows. Because the FRLS-PDA filter can utilize measurement residuals and heading changes to detect the current maneuver of the moving target and determine its

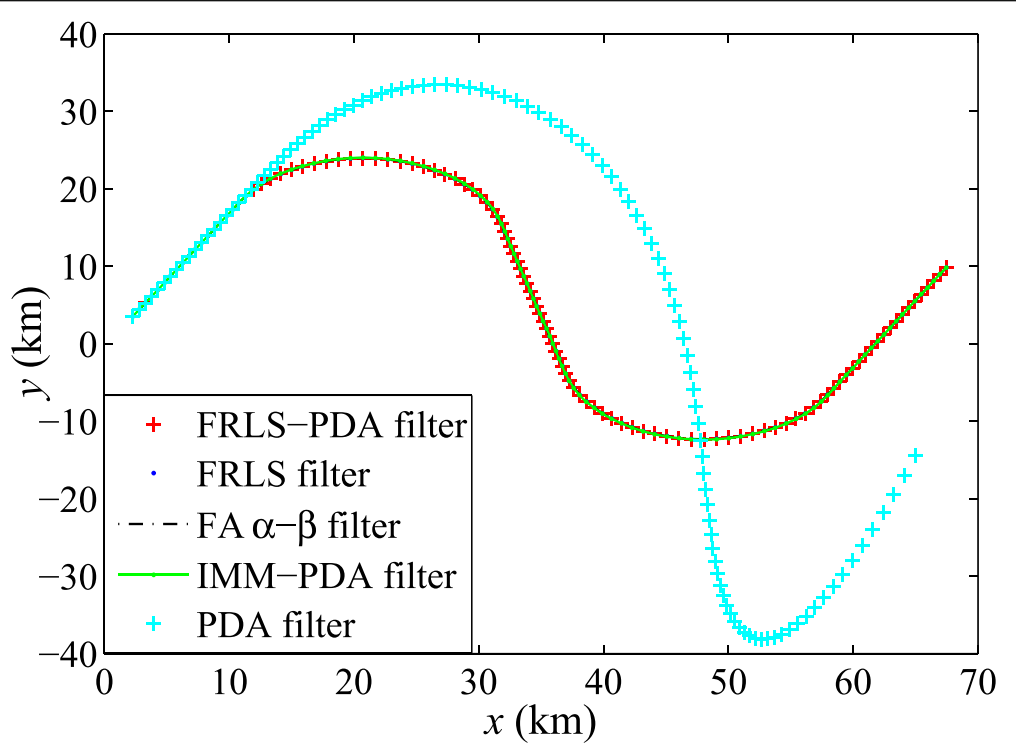

Fig. 4 Tracking results 


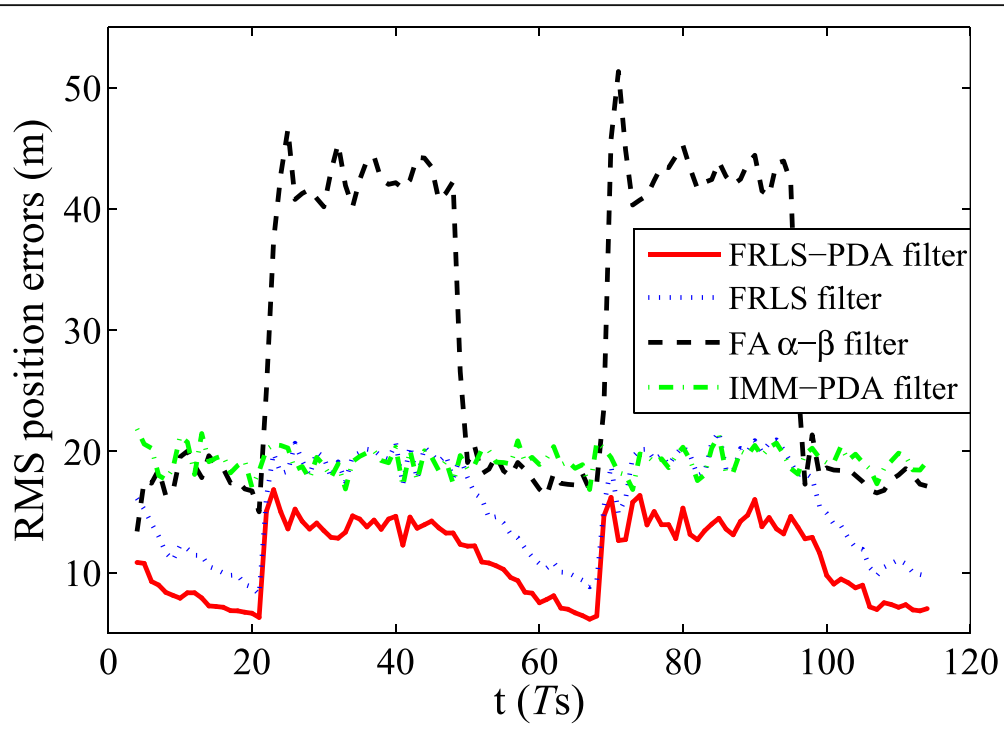

Fig. 5 Estimate errors for four filters

magnitude by the fuzzy system designed, it can obtain the current state timely and accurately. On the other, due to the absence of the prior information of the sub-models, the performance of the IMM-PDA filter is undesirable. The PDA filter performs well in constant velocity phase, but degrades seriously and even diverges in maneuvering phase as shown in Fig. 4. Although the FRLS filter obtains the good performance in constant velocity and maneuvering phase, unfortunately, it cannot be directly utilized in clutter mentioned in Section 1. From the above analysis of the simulation data experiment, it shows that the FRLS-PDA filter can utilize the fused measurements to detect the maneuver in real time and consequently obtain better performance in tracking accuracy than other four filters.

In a word, the proposed filter can provide the following advantages of the PDA algorithm and the FRLS filter: firstly, it can construct a fused measurement through weighting the valid measurements in clutter environments by the association probabilities, which are calculated by the PDA algorithm; secondly, it can relax the restrictive assumption of motion models through using the FRLS filter to estimate the current target state.

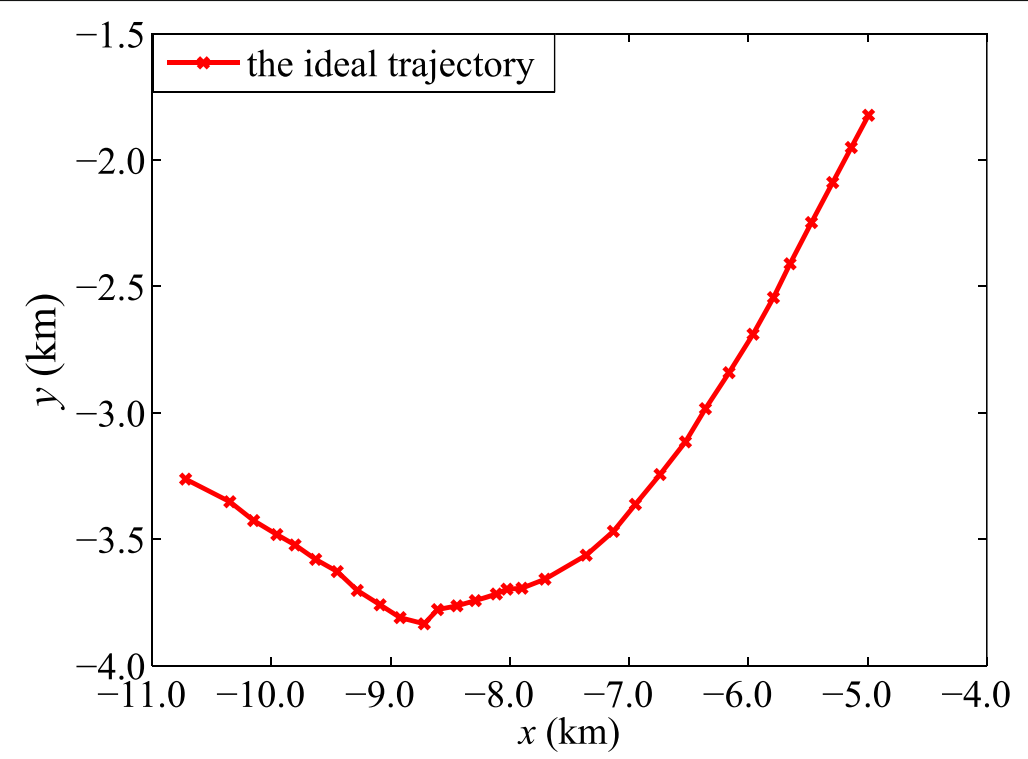

Fig. 6 Ideal trajectory of the target 


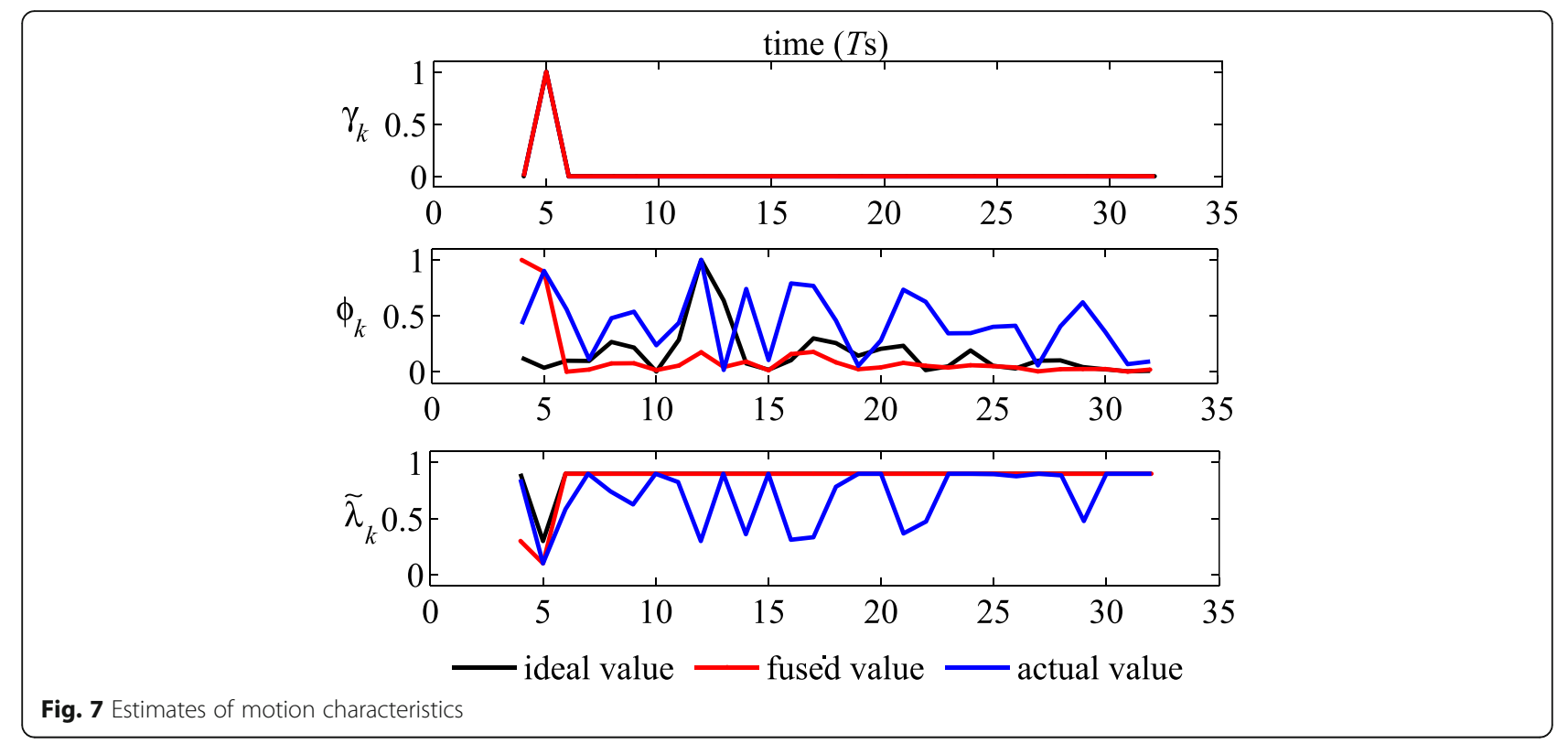

\subsection{Real data experiment}

To illustrate the feasibility of the proposed filter, the real data experiment in [28] is adopted here. The parameters of the clutter model are the same in Section 4.1. The trajectory of the target in Fig. 6 is formed by 32 measurements from some type of single radar and its performance parameters are given as follows: the sampling interval $T=2 \mathrm{~s}$, maximum detection range $r_{\max }=21 \mathrm{~km}$, range error $\sigma_{r}=20 \mathrm{~m}$, and azimuth error $\sigma_{\beta}=0.5^{\circ}$. The initial position of the target is $z_{0}=[-10.72 \mathrm{~km},-$ $3.26 \mathrm{~km}]^{\mathrm{T}}$. Figure 7 shows that the variation curves of $\gamma_{k}, \phi_{k}$, and $\tilde{\lambda}_{k}$ are obtained in the ideal, uncluttered, and cluttered case, respectively. From Fig. $7, \gamma_{k}$ and $\phi_{k}$ can still reflect its changes correctly although the maneuver of the moving target is complicated in the real case. Consequently, the fading factor in uncluttered and cluttered case can be approximated with the values obtained in ideal case. Figures 8 and 9 show the tracking results and RMS position errors by using the above filters for 100 Monte Carlo simulation runs. From Fig. 9, the RMS position errors of the FRLS-PDA filter are smaller than the other three filters. From the real data experiment, it is verified that the proposed filter is feasible to track single maneuvering target in real environment. Therefore, the

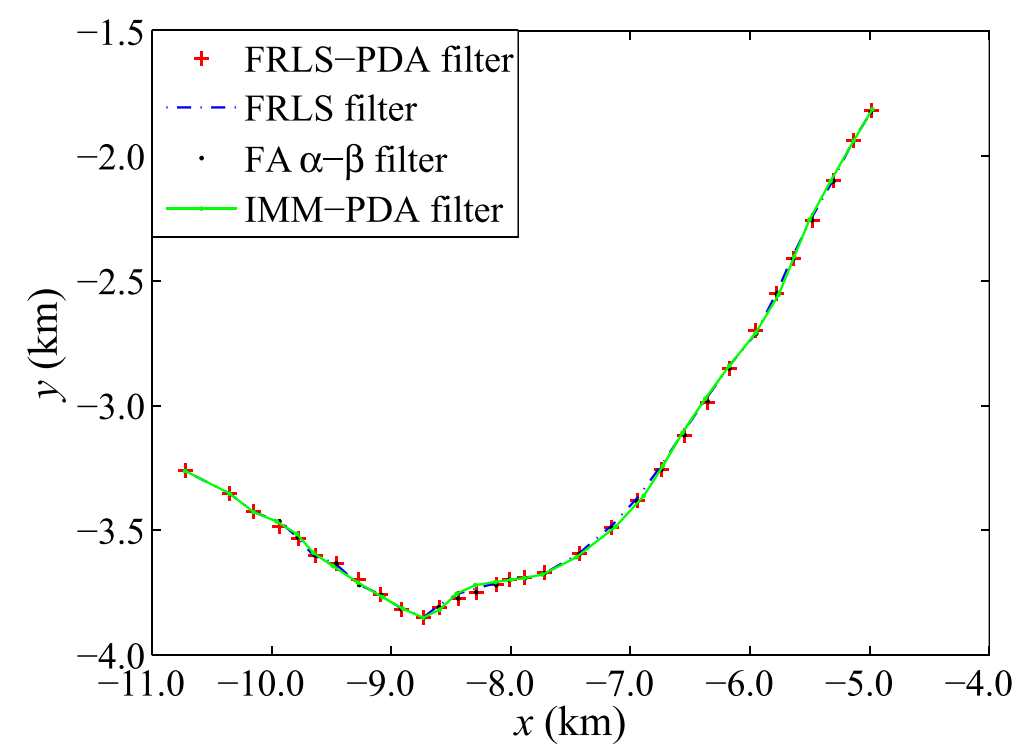

Fig. 8 Tracking results 


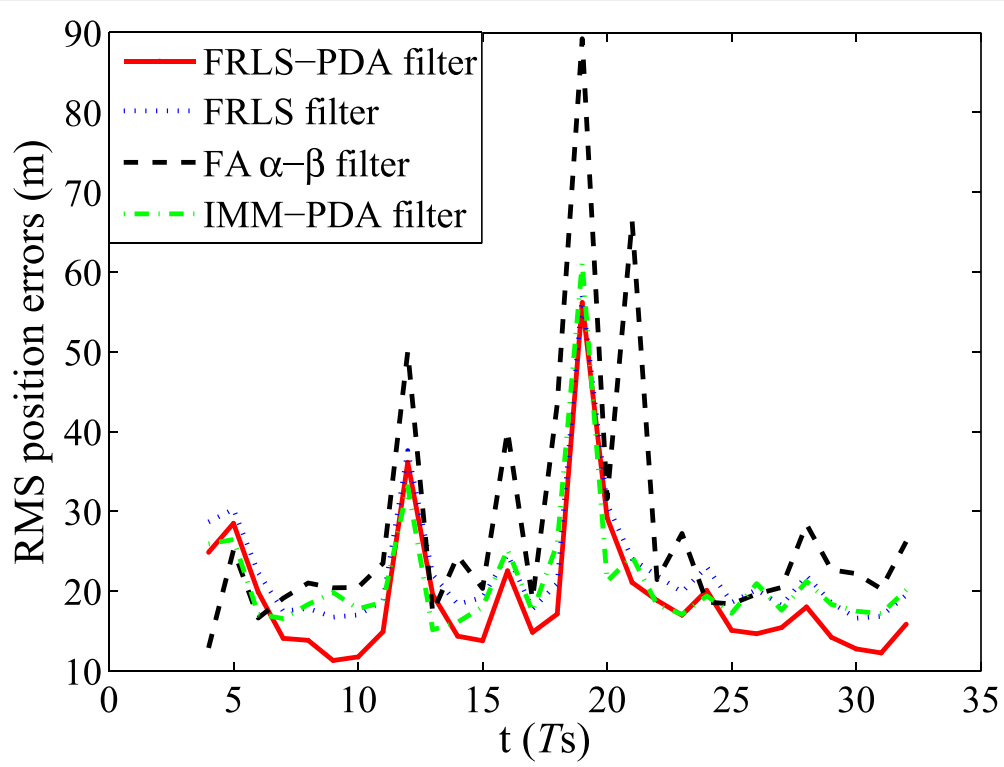

Fig. 9 Estimate errors for four filters

proposed filter can also estimate the maneuver parameter in actual situations and obtain better tracking performance in clutter environments.

\section{Conclusions}

In this paper, a FRLS-PDA filter is proposed based on statistical and fuzzy theories for single MTT in cluttered situations with unknown process noises. In the single MTT process, measurement residuals and heading changes are two important characteristics on maneuvering motion models, which can effectively and exactly reflect maneuvering changes of the moving target. In the proposed filter, the PDA algorithm is utilized to calculate the association probabilities of the measurements that belong to the target, and then these probabilities are used to weighting the valid measurements for constructing the fused measurement at current time. Based on the fused measurement, we can further obtain the current measurement residual and heading change, which acts as the inputs of the fuzzy system to determine the fading factor of the FRLS filter. Hence, the influence of the predicted innovations on the current predicted state can be adjusted through the fuzzy system designed.

The application of the characteristics of motion models into the traditional MTT methods has been proven to be an effective solution to improve their performances in situations with unknown process noises and unknown motion models. The results of two experiments with the simulation data and real data show the effectiveness and feasibility of the proposed filter, and it can realize single MTT effectively. Hence, how to introduce more useful information on maneuvering targets and design the fuzzy rules on the inputs and outputs of the designed fuzzy system is very important in the next work. In real situations, the proposed filter can also be extended to solve the real time MTT problem in sensor networks with multipath or multisource measurements. In addition, the study of the applications of the proposed filter is helpful for solving the MTT problem in sensor network for the future research, including the maneuvering multi-target tracking problems.

\section{Acknowledgements}

This work is supported by the National Natural Science Foundation of China (No. 61603258 and No. 61272034), the Startup Project of Doctor Science Research of Shaoxing University (No. 20145021 and No. 20155015), the Science Project of Shaoxing University (No. 2015LG1006), the Natural Science Foundation of Shenzhen Polytechnic (No. 601522 K21017), the Lanzhou Science and Technology Project (No. 2015-4-3), and the Public Welfare Technology Application Research-Industrial Project of Zhejiang Province (No. 2016C31082).

\section{Competing interests}

The authors declare that they have no competing interests.

\section{Author details}

${ }^{1}$ College of Software Engineering, Lanzhou Institute of Technology, Lanzhou, Gansu 730000, China. ${ }^{2}$ Jožef Stefan International Postgraduate School, Ljubljana 1000, Slovenia. ${ }^{3}$ ATR Key Laboratory, Shenzhen University, Shenzhen, Guangdong 518060, China. ${ }^{4}$ Department of Computer Science and Engineering, Shaoxing University, Shaoxing, Zhejiang 312000, China.

${ }^{5}$ College of Mathematics, Physics and Information Engineering, Jiaxing University, Jiaxing, Zhejiang 314001, China. ${ }^{6}$ Air Defense Forces Academy, Zhengzhou 450052, China.

Received: 1 August 2015 Accepted: 14 September 2016

Published online: 22 September 2016

\section{References}

1. J Zhang, Y Liu, Single maneuvering target tracking in clutter based on multiple model algorithm with Gaussian mixture reduction. J. APPL. Res. Technol. 11(5), 641-652 (2013) 
2. B Khaleghi, A Khamis, FO Karray, SN Razavi, Multisensor data fusion: a review of the state-of-the-art. Inform. Fusion. 14(1), 28-44 (2013)

3. DL Hall, J Llinas, Handbook of Multisensory Data Fusion. LJ Yang, BY Geng, trans. (Publishing House of Electronics Industry, Beijing, 2008)

4. T Sathyan, TJ Chin, S Arulampalam, D Suter, A multiple hypothesis tracker for multitarget tracking with multiple simultaneous measurements. IEEE JSTSP 7(3), 448-460 (2013)

5. J Chen, H Leung, T Lo, J Litva, M Blanchette, A modified probabilistic data association filter in a real clutter environment. IEEE Trans. Aero. Elec. Sys. 32(1), 300-313 (1996)

6. X Jiang, K Harishan, R Tharmarsa, T Kirubarajan, T Thayaparan, Integrated track initialization and maintenance in heavy clutter using probabilistic data association. Signal Process. 94, 241-250 (2014)

7. ZX Liu, WX Xie, JX Huang, A new probabilistic data association filter based on probability theory. J. Elec. Inform. Tech. 31(7), 1641-1644 (2009)

8. JA McGee, TE Luginbuhl, JH Dibiase, PL Ainsleigh, A modified PDAF algorithm for measurement covariances that vary within a time scan. IEEE Trans. Aero. Elec. Sys. 48(1), 906-912 (2012)

9. S Vasuhi, $V$ Vaidehi, Target tracking using interactive multiple model for wireless sensor network. Inform. Fusion. 27, 41-53 (2015)

10. M Compare, P Baraldi, P Turati, E Zio, Interacting multiple-models, state augmented particle filtering for fault diagnostics. Probabilist. Eng. Mech. 40, 12-24 (2015)

11. XJ Jin, GD Yin, Estimation of lateral tire-road forces and sideslip angle for electric vehicles using interacting multiple model filter approach. J. Franklin I. 352(2), 686-707 (2015)

12. K Jo, J Kim, M Sunwoo, Real-time road-slope estimation based on integration of onboard sensors with GPS using an IMMPDA filter. IEEE Trans. Intell. Transp. Sys. 14(4), 1718-1732 (2013)

13. C Scheel, SP Linder, JR Zeidler, Tracking highly maneuverable targets with unknown behavior. P. IEEE. 92(3), 558-574 (2004)

14. PDZ Varcheie, GA Bilodeau, Adaptive fuzzy particle filter tracker for a PTZ camera in an IP surveillance system. IEEE T. Instrum. Meas. 60(2), 354-371(2011)

15. ZT Hu, CL Fu, XX Liu, Maneuvering multi-target tracking algorithm based on modified generalized probabilistic data association. Engineering. 3, 1155-1160 (2011)

16. YL Wei, JB Qiu, HR Karimi, M Wang, A new design of $\mathrm{H}_{\infty}$ filter for continuous-time Markovian jump systems with time-varying delay and partially accessible mode information. Signal Process. 93, 2392-2407 (2013)

17. YL Wei, JB Qiu, RK Hamid, M Wang, Model reduction for continuous-time Markovian jump systems with incomplete statistics of mode information. Int. J. Syst. SCI. 45(7), 1496-1507 (2014)

18. YL Wei, M Wang, JB Qiu, New approach to delay-dependent $H_{\infty}$ filtering for discrete-time Markovian jump systems with time-varying delay and incomplete transition descriptions. IET Control Theory A. 7(5), 684-696 (2013)

19. LQ Li, WX Xie, Intuitionistic fuzzy joint probabilistic data association filter and its application to multitarget tracking. Signal Process. 96, 433-444 (2014)

20. LQ Li, WX Xie, Bearings-only maneuvering target tracking based on fuzzy clustering in a cluttered environment. AEU-Int. Electron. C. 68(2), 130-137 (2014)

21. PF Li, JP Yu, LQ Li, Maneuvering target tracking based on fuzzy adaptive $\alpha-\beta$ filter. Sys. Eng. Elec. 30(11), 2138-2141 (2008)

22. LX Wang, A Course in Fuzzy Systems and Control. Y. J. Wang, trans. (Tsinghua University Press, Beijing, 2003)

23. E Fan, WX Xie, ZX Liu, Reliability-weighted nearest neighbor track association in sensor network. Sensor Lett. 12(2), 319-324 (2014)

24. I Turkmen, IMM fuzzy probabilistic data association algorithm for tracking maneuvering target. Expert Syst. Appl. 34(2), 1243-1249 (2008)

25. M Oussalah, J De Schutter, Hybrid fuzzy probabilistic data association filter and joint probabilistic data association filter. Inform. Sciences. 142(1), 195-196 (2002)

26. HS Son, JB Park, YH Joo, Fuzzy c-means-based intelligent tracking algorithm for an underwater manoeuving target. IET Radar Sonar Nav. 8(9), 623-634 (2014)

27. HS Son, JB Park, YH Joo, Fuzzy c-means clustering-based smart tracking model for three-dimensional manoeuving target including unknown acceleration input. IET Radar Sonar Nav. 7(6), 623-634 (2013)

28. E Fan, WX Xie, ZX Liu, Maneuvering target tracking using fuzzy logic-based recursive least squares filter. EURASIP J. Adv. Sig. Pr. 2014(1), 1-9 (2014)
29. YM Zhu, Efficient recursive state estimator for dynamic systems without knowledge of noise covariances. IEEE Trans. Aero. Elec. Sys. 35(1), 102-114 (1999)

30. MZA Bhotto, A Antoniou, New improved recursive least-squares adaptive-filtering algorithms. IEEE T. on Circuits-I 60(6), 1548-1558 (2013)

31. MK Awad, KT Wong, Recursive least-squares source tracking using one acoustic vector sensor. IEEE Trans. Aero. Elec. Sys. 48(4), 3073-3083 (2012)

\section{Submit your manuscript to a SpringerOpen ${ }^{\circ}$ journal and benefit from:}

- Convenient online submission

- Rigorous peer review

- Immediate publication on acceptance

- Open access: articles freely available online

- High visibility within the field

- Retaining the copyright to your article

Submit your next manuscript at springeropen.com 